\title{
Intuitive Hand Teleoperation by Novice Operators Using a Continuous Teleoperation Subspace
}

\author{
Cassie Meeker $^{1}$, Thomas Rasmussen ${ }^{1}$ and Matei Ciocarlie ${ }^{1}$
}

\begin{abstract}
Human-in-the-loop manipulation is useful in when autonomous grasping is not able to deal sufficiently well with corner cases or cannot operate fast enough. Using the teleoperator's hand as an input device can provide an intuitive control method but requires mapping between pose spaces which may not be similar. We propose a low-dimensional and continuous teleoperation subspace which can be used as an intermediary for mapping between different hand pose spaces. We present an algorithm to project between pose space and teleoperation subspace. We use a non-anthropomorphic robot to experimentally prove that it is possible for teleoperation subspaces to effectively and intuitively enable teleoperation. In experiments, novice users completed pick and place tasks significantly faster using teleoperation subspace mapping than they did using state of the art teleoperation methods.
\end{abstract}

\section{INTRODUCTION}

In unstructured environments that require unscripted and complex manipulation, it is often useful to utilize humanin-the-loop manipulation in lieu of autonomous grasping. When a large number of possible scenarios and objects can be encountered, human cognition makes decisions faster and deals with corner cases better than autonomous algorithms.

Teleoperation which harvests the movement of the teleoperator's hand to control a robot hand can provide an intuitive and user-friendly interface [1]. This type of teleoperation uses hand motions which are already familiar to the user, instead of requiring knowledge of external control hardware. However, teleoperation based on the operator's hand movement requires mapping between the pose spaces of the two hands of interest. Intuitive teleoperation mappings are desirable because they help teleoperators, particularly novice teleoperators, to complete tasks in a safe and timely manner.

Robot hand designs which are fully-actuated and anthropomorphic allow for an intuitive mapping between hands and thus are intuitive for a human to teleoperate; however, the hardware tends to be fragile and expensive. In contrast, non-anthropomorphic hands have proven to be robust and versatile in unstructured environments. However, finding an easy or intuitive mapping between the human hand and a non-anthropomorphic robot hand can be difficult, due to the different joints, different axes, different numbers of fingers, or any number of dissimilarities between the hands.

In this paper, we seek to create an intuitive mapping between the human hand and a fully actuated non-

\footnotetext{
* This work was supported in part by the ONR Young Investigator Program award N00014-16-1-2026.

${ }^{1}$ Department of Mechanical Engineering, Columbia University, New York, NY 10027, USA

\{com2144, matei.ciocarlie\}@columbia.edu

\{tomras12\}@gmail.com
}

anthropomorphic robot hand that enables effective real-time teleoperation for novice users.

The method we propose uses a subspace relevant to teleoperation as an intermediary between the pose spaces of two different hands. Our method enables teleoperation by projecting the pose of the master hand into the defined teleoperation subspace, which it shares with the slave hand, and then projecting from the teleoperation subspace into the pose space of the slave hand.

Unlike traditional pose mapping, we avoid unexpected movements by using a continuous subspace as a basis for mapping instead of interpolation between discrete poses. Our mapping is independent of the master-slave pairing, so the mapping between teleoperation subspace and pose space of a robot does not have to be redefined with every new human teleoperator. Furthermore, the teleoperation subspace is low dimensional, which allows for the future possibility of simple control mechanisms, such as cursor control or electromyography (EMG) based controls, although these are not explored in this work.

Our main contributions are as follows: we introduce a continuous, low-dimensional teleoperation subspace as an intuitive way to map human to robot hand poses for teleoperation. We posit that this method allows for intuitive teleoperation as long as both the master and the slave hand poses can be projected into this subspace. We provide an empirical method for achieving this projection, and experimentally prove that it is effective and intuitive using a robot hand with highly non-anthropomorphic kinematics. Our method allows novice teleoperators to pick and place objects significantly faster than state of the art teleoperation mapping methods.

\section{RELATED WORK}

Conventional teleoperation methods are divided into three main categories: joint mapping, fingertip mapping, and pose mapping.

Joint mapping (also called joint-to-joint mapping) is used when the slave hand has similar kinematics to the human controller [2]. If the human and robot joints have a clear correspondence, the human joint angles can be imposed directly onto the robot joints with little or no transformation [3]. This mapping is most useful for power grasps [4], and is limited if the robot hand is non-anthropomorphic.

Fingertip mapping (also called point-to-point mapping) is the most common teleoperation mapping method. Forward kinematics transform human joint angles into Cartesian fingertip positions. These undergo scaling to find the desired robot Cartesian fingertip positions and then inverse 
kinematics determine robot joint angles. This mapping is useful for precision grasps [4]. When the robot has less than five fingers, the extra human fingers are ignored [5]. Error compensation can find the closest fit when human and robotic workspaces are incompatible [6]. However, teleoperation using this method is difficult when the workspaces of the human and robotic fingers are not similar.

An alternative to fingertip mapping is virtual object mapping, which uses the relative distances between fingertips. Fingertip mapping uses the distance between the master fingertips and inverse kinematics to calculate joint angles that place the slave fingertips at the same relative distance. This method can be used in both 2-D [7] and 3-D [8][9] grasping scenarios. The relative distances between fingertips are often calculated based on Cartesian fingertip positions, so virtual object mapping is similar to fingertip mapping. Virtual object mapping is useful for tasks which involve dexterous manipulation [10]; however it is often unsuitable for tasks which require different grasp types or which involve irregularly shaped objects. This mapping was unsuitable for our experiments because of the variety of objects we wished to pick and place. Virtual object mapping can generalize to virtual objects of any shape [11], assuming that contact points for the slave and master hands can be tracked. This is difficult without haptic devices, which we did not use in this work.

Pose mapping attempts to replicate the pose of the human hand with a robot hand, which is appealing because, unlike fingertip and joint mapping, it attempts to interpret the function of the human grasp rather than replicate hand position. Pao and Speeter define transformation matrices relating human and robot poses using least squared error compensation when this transformation is not exact [12]. Others use neural networks to identify the human pose and map the pose to a robot either through another neural network [13] or pre-programmed joint-to-joint mapping [14]. Outside of a discrete set of known poses, pose mapping can lead to unpredictable hand motions and is usually used when only simple grasping poses are required. Furthermore, the above mappings that use neural networks require classification of the human hand pose before it is mapped to the robotic hand. If this classifier misidentifies the human pose, the robot hand will move in undesirable ways. Our method also attempts to replicate hand shape, rather than fingertip or joint positions, making it most similar to pose mapping, but we do not require discrete classification of human pose before mapping.

This paper introduces a low-dimensional mapping. Other methods that define grasping in a low dimensional space include postural synergies, which are low dimensional and continuous [15]. Just as synergies move the description of human hand position from discrete, static poses [16] into a continuous space, we seek to allow pose mapping between the human and robotic hand to be continuous instead of interpolating between discrete poses.

In the field of autonomous grasp planning, finding synergies for robotic hands based on human synergies can inform grasp planning [17][18][19]. Other works show synergies

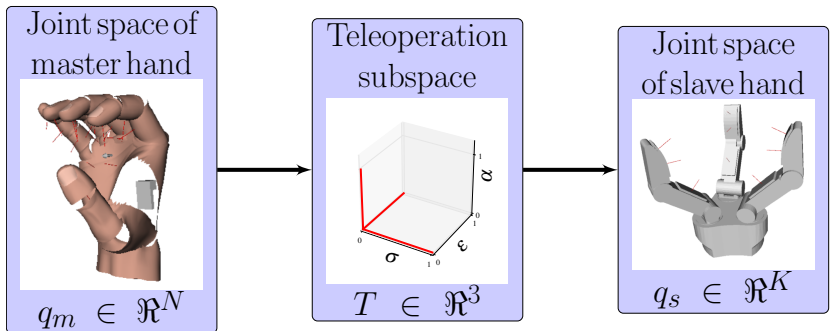

Fig. 1. Steps to enable real time teleoperation using teleoperation subspace

to also be an effective control for teleoperation. Jenkins demonstrated a low dimensional control which could potentially be used to teleoperate robotic systems with cursor control [20]. To our knowledge, this has only been tested in simulation. Brygo, et al. translated postural synergies from joint space to fingertip Cartesian space to control teleoperation [21]. This work only considers the first postural synergy, and is most appropriate for underactuated hands. Kim, et al. demonstrated a synergy level controller which uses multiple postural synergies to enable teleoperation [22]. This method calculates the synergies of the robot hand through pose mapping, which could have discontinuities. They calculated robotic synergy coefficients based, in part, on the rate of change for each synergy coefficient. Our method does not have discontinuities and does not have a temporal component.

Other works use low dimensional latent variables which are not based on synergies to approximate human poses in non-anthropomorphic models. These latent variables have enabled both the animation of non-anthropomorphic creatures [23] and teleoperation. Gaussian process latent variable models (GP-LVM) can enable teleoperation of humanoid robots. In some formulations, the latent space changes with every different master-slave pairing [24]. In other formulations, multiple robots and a human share the same latent space [25]. These latent variable models require the user to generate observations where the master and slave poses are correlated in order to train the GP-LVM model. A given GP-LVM model requires the user to generate tens, and even up to hundreds of correlated poses for training. Although we are inspired by a similar desire to find shared subspaces between robotic and human hands, our method only requires the user to generate one correlated pose. We also require two other vectors for each hand, but we outline a simple way to calculate those vectors that does not require consideration of the master-slave pairing.

\section{Teleoperation Subspace}

As a general concept, we posit that, for many hands, a three dimensional space $T$ isomorphic to $\Re^{3}$ can encapsulate the range of movement needed for teleoperation. The three basis vectors of $T$ have an intuitive correspondence with hand shape. One corresponds with how far apart the fingers are spread, another with the size of the object a hand can grasp, and another with how curled the fingers are. We refer to these basis vectors as $\alpha, \sigma$, and $\epsilon$, respectively. 

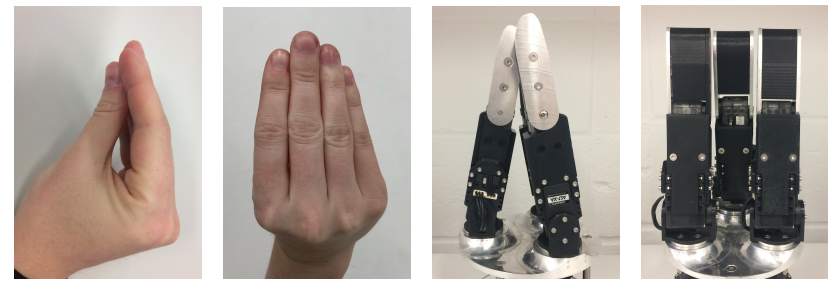

Origin pose of the human hand. Origin pose of the robotic hand. Fig. 2. Origin poses of two example hands.

We chose these bases using on intuition, guided by Santello's research of postural synergies [15]. Since Santello, et al. used principle component analysis (PCA), a linear dimension reduction method, to find postural synergies, we also assume that mapping between pose space and teleoperation subspace is linear.

We assume that many hands will be able to project their pose spaces into $T$. If this projection is possible, $T$ is embedded as a subspace in the pose space of the hand. $T$ is thus a subspace "shared" by all hands that can project their pose space into $T$. If the user can construct a projection matrix which projects pose space to teleoperation subspace in a meaningful way, our method will enable teleoperation. Experimentally, we prove that $T$ is relevant for teleoperation for at least the human hand and a non-anthropomorphic robot hand, similar to the Schunk SDH. We theorize that $T$ is also relevant to teleoperation for other hands.

To teleoperate using $T$, there are two steps:

1) Given joint values of the master hand $h_{m}$, find the equivalent pose $t$ in teleoperation subspace $T$

2) Given $t$ computed above, find the joint values of the slave hand $h_{s}$, and move $h_{s}$ to these values

In order to enact the teleoperation steps, we must first project between $T$ and the relevant pose spaces.

\section{A. Projecting between Pose Space and Teleoperation Sub- space}

We define an empirical projection method from pose space to teleoperation subspace. For a given hand with $N$ joints, projecting from joint space $q \in \Re^{N}$ (here we use pose space and joint space interchangeably) into teleoperation subspace $T$ requires an origin pose $o \in \Re^{N}$, a projection matrix $A \in$ $\Re^{N \times 3}$, and a scaling factor $\delta \in \Re^{3}$.

1) Origin $o$ : To project between joint space and $T$, we require a hand-specific, "neutral" origin pose $o \in \Re^{N}$.

$$
o=\left[o_{1}, o_{2}, \ldots, o_{N}\right]
$$

This represents a hand position which will standardize the data as we project between joint space and $T$. The origin pose of the master is arbitrary; however, it is crucial that the origin pose of the slave corresponds to the master's origin (i.e. the two hands should assume approximately the same shape while positioned at their respective origins). Figure 2 shows the pose we chose for the human hand and the custombuilt robotic hand in our experiments.
2) Projection Matrix A: The projection matrix $A \in \Re^{N \times 3}$ is hand specific and consists of three basis vectors $\psi \in \Re^{N}$. Each $\psi$ is a projection of one of $T$ 's basis vectors in pose space. The $\psi$ s which correspond to $\boldsymbol{\alpha}, \boldsymbol{\sigma}$, and $\boldsymbol{\epsilon}$ are referred to as $\psi_{\alpha}, \psi_{\sigma}$, and $\psi_{\epsilon}$, respectively.

$$
\begin{array}{r}
A=\left[\psi_{\alpha}, \psi_{\sigma}, \psi_{\epsilon}\right] \\
\psi_{\alpha}=\left[\psi_{\alpha 1}, \psi_{\alpha 2}, \ldots, \psi_{\alpha N}\right]^{\top} \\
\psi_{\sigma}=\left[\psi_{\sigma 1}, \psi_{\sigma 2}, \ldots, \psi_{\sigma N}\right]^{\top} \\
\psi_{\epsilon}=\left[\psi_{\epsilon 1}, \psi_{\epsilon 2}, \ldots, \psi_{\epsilon N}\right]^{\top}
\end{array}
$$

For each joint, the user determines the basis vector in $T$ to which a joint has the most intuitive correspondence, and then sets that element equal to 1 in the $\psi$ which is a projection of that basis vector. The user sets joints which adduct the fingers to 1 in $\psi_{\alpha}$, joints which open the hand to 1 in $\psi_{\sigma}$, and joints which curl the fingers to 1 in $\psi_{\epsilon}$. This is a winner take all approach, so a joint may only contribute to a single $\psi$. This is most relevant for underactuated hands where a single joint could simultaneously serve to open the hand and spread the fingers, but can only be non-zero in one $\psi$. After the $\psi$ s are built, the user normalizes each $\psi$ to create $A$.

As an illustrative example, we build $A$ for the robotic slave hand used in our experiments. The robotic hand has eight joints - the thumb $(f 0)$ has a proximal and distal joint, and the two opposing fingers ( $f 1$ and $f 2$ ) have proximal, distal and adduction joints. We define the vector of joints as:

$j=\left[f 0_{\text {prox }}, f 0_{\text {dis }}, f 1_{\text {ad }}, f 1_{\text {prox }}, f 1_{\text {dis }}, f 2_{\text {ad }}, f 2_{\text {prox }}, f 2_{\text {dis }}\right]$

For this hand, joints which spread the fingers are the adductors at $j[2]$ and $j[5]$. These joints have the most intuitive correspondence to $\alpha$ so they are set to 1 in $\psi_{\alpha}$ :

$$
\psi_{\alpha}=[0,0,1,0,0,1,0,0]
$$

Changing the values at the proximal joints allows the hand to grasp objects of varying sizes, meaning they correspond to $\sigma$. Since $j[0], j[3]$ and $j[6]$ represent the proximal joints, we set the values at these indices as 1 in $\psi_{\sigma}$ :

$$
\psi_{\sigma}=[1,0,0,1,0,0,1,0]
$$

For the robotic hand, changing the values at the distal joints will curl the fingers. The distal joints correspond to $j[1], j[4]$ and $j[7]$, so we set the values at these indices as 1 in $\psi_{\epsilon}$ :

$$
\psi_{\epsilon}=[0,1,0,0,1,0,0,1]
$$

Finally, we normalize the above $\psi$ s in order to create $A$.

Even though our method requires user intuition to build the projection matrix $A$, the process of building $A$ as described above is fairly simple. Experimentally, we prove that these basic calculations are sufficient to meaningfully project pose space into $T$ in a way that enables teleoperation.

3) Scaling Factor $\delta$ : We wish to normalize such that any configuration in pose space will project to a pose in $T$ whose value is less than or equal to 1 along each of the basis vectors. We therefore require a scaling factor $\delta \in \Re^{3}$ to normalize the projection:

$$
\delta=\left[\delta_{\alpha}, \delta_{\sigma}, \delta_{\epsilon}\right]
$$




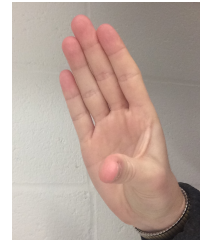

Pose for maximum along $\boldsymbol{\sigma}$, minimum along $\boldsymbol{\alpha}$.

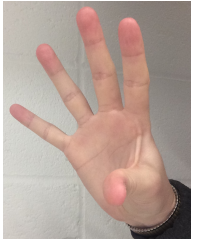

Pose for maximum along $\alpha$.

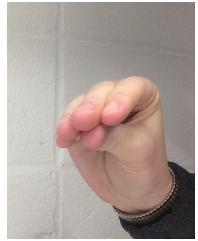

Pose for minimum along $\boldsymbol{\epsilon}$, and minimum along $\boldsymbol{\sigma}$.

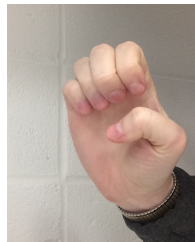

Pose for maximum along $\epsilon$.
Fig. 3. Poses required from the human user to demonstrate maximum and minimum of the unscaled teleoperation subspace projection.

To calculate $\delta$, we evaluate poses which illustrate the extrema of the hand's kinematic limits along the basis vectors. For example, the maximum and minimum values along $\boldsymbol{\sigma}$ are illustrated by projecting poses where the hand is holding the largest object possible and the smallest object possible from pose space into $T$. It is up to the user to determine poses which illustrate the full range of values for each basis vector. Figure 3 shows the poses which demonstrate these ranges for the human hand.

Once we select the illustrative poses for the hand, we project these poses from pose space into $T$ using $t=$ $(q-o) \cdot A$, where $t \in T$. From this set of poses in $T$, we find the minimum and maximum values along each axis. Along $\boldsymbol{\alpha}$, the minimum and maximum are referred to as $\alpha_{\min }$ and $\alpha_{\max }$, respectively. From these values, we calculate $\delta_{\boldsymbol{\alpha}}$ as:

$$
\begin{gathered}
\alpha_{\text {range }}=a b s\left(\alpha_{\max }\right)+a b s\left(\alpha_{\min }\right) \\
\delta_{\boldsymbol{\alpha}}= \begin{cases}0 & \text { if } \alpha_{\text {range }}=0 \\
1 / \alpha_{\text {range }} & \text { otherwise }\end{cases}
\end{gathered}
$$

Finding $\delta_{\boldsymbol{\sigma}}$ and $\delta_{\boldsymbol{\epsilon}}$ uses the same calculation.

$\delta$ normalizes the projection from pose space to $T$; however, to project from $T$ back to pose space, we require an inverse scaling factor $\delta^{*}$ :

$$
\begin{array}{r}
\delta^{*}=\left[\delta_{\alpha}^{*}, \delta_{\sigma}^{*}, \delta_{\epsilon}^{*}\right] \\
\delta_{\alpha}^{*}= \begin{cases}0 & \text { if } \delta_{\alpha}=0 \\
1 / \delta_{\alpha} & \text { otherwise }\end{cases}
\end{array}
$$

where we find $\delta_{\sigma}^{*}$ and $\delta_{\epsilon}^{*}$ with similar calculations.

4) A Complete Projection Algorithm: To project between teleoperation subspace $T$ and joint space $q$, we use the handspecific matrix $A$, the origin $o$, and the scaling factor $\delta$ :

$$
\begin{array}{r}
t=((q-o) \cdot A) \odot \delta \\
q=\left(\left(t \odot \delta^{*}\right) \cdot A^{\top}\right)+o
\end{array}
$$

where, $\odot$ represents element-wise multiplication.

To use $T$ for teleoperation, Eq. (15) projects the master hand's pose space into the shared teleoperation subspace and then Eq. 16 projects from the shared teleoperation subspace into the slave hand's pose space.

So, given the joint angles of the master hand, we are able to calculate the joint angles of the slave hand using:

$$
q_{s}=\left(\left(\left(q_{m}-o_{m}\right) \cdot A_{m}\right) \odot \delta_{m} \odot \delta_{s}^{*}\right) \cdot A_{s}^{\top}+o_{s}
$$

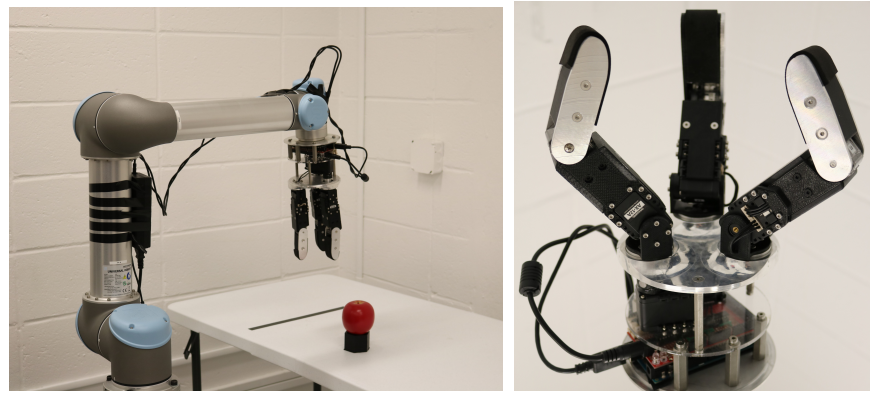

Fig. 4. Left: experimental set-up with UR5 arm, robotic hand and table Right: close-up of the custom robotic hand used in experiments.

\section{EXPERIMENTS}

To show that the proposed method is intuitive for novice users, experiments were performed with five healthy subjects. Two subjects were female, three subjects were male, all were aged between 23 and 28, and all were novice robot teleoperators. Subjects gave their informed consent and the study was approved by the Columbia University IRB.

We compared our teleoperation method with two state of the art teleoperation techniques - joint mapping and fingertip mapping. These methods were chosen from the state of the art as being the most applicable to our problem: intuitive for novice users, and able to use different grasping types (power and precision, for example) to grasp objects of various shapes and sizes. We used time to complete a pick and place task as a metric for usability and intuitiveness.

\section{A. Experimental Setup}

The slave hand of our teleoperation system is a custombuilt robotic hand with kinematics similar to the Schunk SDH. It has a thumb finger and two opposing fingers; all three fingers have two links. The hand is fully actuated and the two opposing fingers adduct independently.

We attached the slave hand to a Universal Robot (UR5) arm. The UR5 stands in front of a table where the grasping objects are placed one at a time during testing. Figure 4 shows our setup, and a close-up of the slave hand.

The novice teleoperator stands next to the robot and teleoperates based on visual feedback. Attached to the user's hand is an Ascension 3D Guidance trakSTAR ${ }^{\mathrm{TM}}$ system, which tracks hand position and orientation. The UR5 follows the position and the orientation of the trakSTAR with a cartesian controller. We control the robotic hand using either joint, fingertip or teleoperation subspace mapping.

Subjects participated in two testing sessions. During the first session, we presented the subjects with the subspace and joint mapping teleoperation methods. During a second session, we presented subjects with the fingertip mapping control. The order in which the joint mapping and subspace mappings were presented to the users was randomized; however, all subjects performed the fingertip mapping last. Nominally, subjects should have been able to use the fingertip mapping faster because they were already familiar with the arm and the hand by the second session.

During the first session, the teleoperator wore a data glove (a Cyberglove) with a trakSTAR sensor attached to the back 


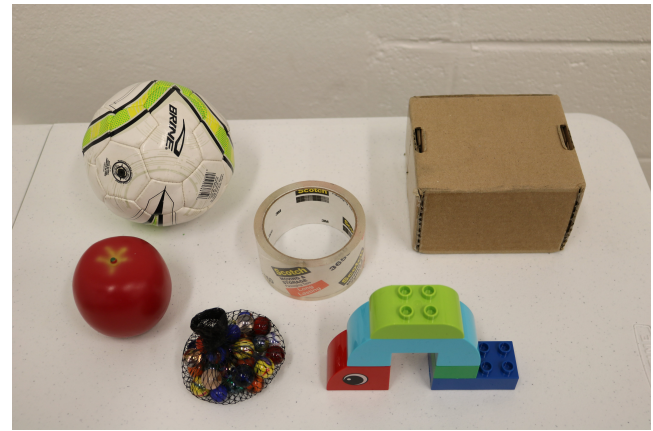

Fig. 5. Objects used in pick and place tasks

of the hand. The Cyberglove provided the joint angles of the human hand to the mapping control method. The trakSTAR provided hand position and orientation to the UR5 controller.

During the second session, the teleoperator wore a trakSTAR sensor attached to the back of the hand, as well as additional sensors attached to three of the fingers. All four sensors were used for the fingertip mapping and the sensor on the back of the hand again provided hand position and orientation to the UR5 controller.

\section{B. Testing}

We placed a series of objects on the table one at a time and asked subjects to pick them up and move them across a line 0.3 meters away. Shorter objects were placed on a stand so as to facilitate easier grasping with the large robotic fingers. The objects for the pick and place tasks were: a box, a ball, a stack of Legos, a roll of tape, a plastic apple and a mesh bag of marbles (Figure 5). We selected these object to illustrate a variety of grasping types. We did not instruct the users how they should grasp the objects. After the subject placed an object in the designated area, we reset the UR5 to a neutral position before the next pick and place task.

At the beginning of each session, the subject was given three minutes to move the arm, but not the hand. We did not want the user's unfamiliarity with the arm to bias the results towards the second control method during the first session. Furthermore, the first object the user attempted to grasp (the box) was labeled as a training object and not included in the evaluation.

We explained the mapping method to the teleoperator immediately before they were asked to pick and place objects using that control.

\section{Baseline Comparison: Fingertip Mapping}

We chose fingertip mapping as a comparison because it is applicable to precision grasps, particularly with smaller objects. The fingertip mapping method was designed as follows: first, we found the cartesian positions of the thumb, index, and ring fingers of the human hand with respect to the wrist by attaching trakSTAR sensors to each of the listed fingers and to the back of the wrist. We calculated transforms between the wrist and finger sensors to find the finger position in the hand frame. We multiplied these positions by a scaling factor of 1.5 , the ratio between an average human finger and one of the robotic fingers. We rotated
TABLE I

MAPPING FROM THE CYBERgLOVE TO THE CUSTOM Robotic HAND

\begin{tabular}{cc|cc}
\multicolumn{2}{c|}{ Cyberglove Sensor } & \multicolumn{2}{c}{ Robotic Hand Joints } \\
Joint & Name & Label & Name \\
Label & Thumb adduction & 1 & Thumb proximal flexion \\
\hline $\mathrm{a}$ & Thumb distal flexion & 2 & Thumb distal flexion \\
$\mathrm{b}$ & Index/Middle adduction & 3 & Finger 1 adduction \\
$\mathrm{e}$ & Index proximal flexion & 4 & Finger 1 proximal flexion \\
$\mathrm{c}$ & 5 & Finger 1 distal flexion \\
$\mathrm{d}$ & Index medial flexion & 5 & Finger 2 adduction \\
$\mathrm{e}$ & Index/Middle adduction & 6 & Finger 2 proximal flexion \\
$\mathrm{f}$ & Middle proximal flexion & 7 & Finger 2 distal flexion \\
$\mathrm{g}$ & Middle medial flexion & 8 &
\end{tabular}
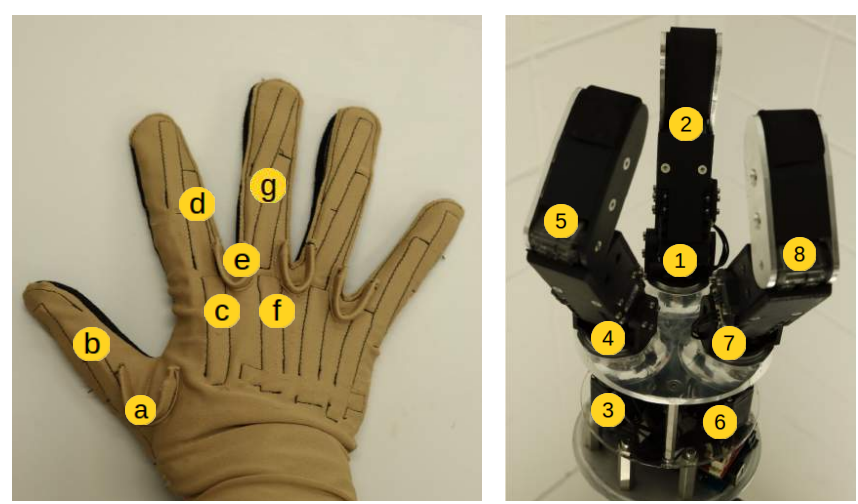

Fig. 6. Joint labels for joint mapping

the positions from the human hand frame into the robotic hand frame. We translated the coordinates from the robotic hand frame into the finger frame to find the desired robotic fingertip positions. Finally inverse kinematics determined the joint angles which placed the fingertips at these positions. This process is documented elsewhere [19].

\section{Baseline Comparison: Joint Mapping}

We chose joint mapping as the second comparison method because it is applicable to power grasps. We also predicted that explicit control over individual joints of the robotic fingers would be intuitive for novice users. Rosell, et al. teleoperated the Schunk SDH using joint mapping by assigning the joints of all fingers of the robot hand to correspond the joints of one finger of the human hand, with finger adduction set to a constant value [26]. We instead chose to map each joint of the robot finger to a separate joint of the human hand so as not to limit the subject's ability to perform different grasp types.

To implement joint mapping, we assigned each of the robot joints to a corresponding human hand joint. This mapping can be found in Table 1 and Figure 6 . We rotated the joint angles of the human hand received from the Cyberglove so they aligned with the robotic hand, and set the joints of the robot hand to these rotated angles.

Preliminary tests showed teleoperation is difficult if the robot thumb's proximal joint maps to the human thumb's metacarpophalangeal (MCP) joint. We therefore mapped the robot thumb's proximal joint to the human thumb's adductor. 
TABLE II

AVERAGE TIME (IN SECONDS) TO PICK AND PLACE COMPLETION

\begin{tabular}{c|ccccc|c} 
& \multicolumn{6}{|c}{ Object } \\
$\begin{array}{c}\text { Mapping } \\
\text { Method }\end{array}$ & Ball & Legos & Apple & Tape & Marbles* & Average \\
\hline Fingertip & 64.67 & 31.83 & 37.05 & 47.02 & 153.6 & 62.27 \\
Joint & 23.09 & 45.43 & 51.53 & 27.25 & 157.21 & 56.66 \\
Subspaces & $\mathbf{1 9 . 2 2}$ & $\mathbf{2 2 . 2 7}$ & $\mathbf{2 3 . 9 7}$ & $\mathbf{2 2 . 6 3}$ & $\mathbf{3 3 . 4}$ & $\mathbf{2 7 . 5 2}$
\end{tabular}

* denotes that the average was calculated with a smaller sample size for some of the mapping methods of that object.

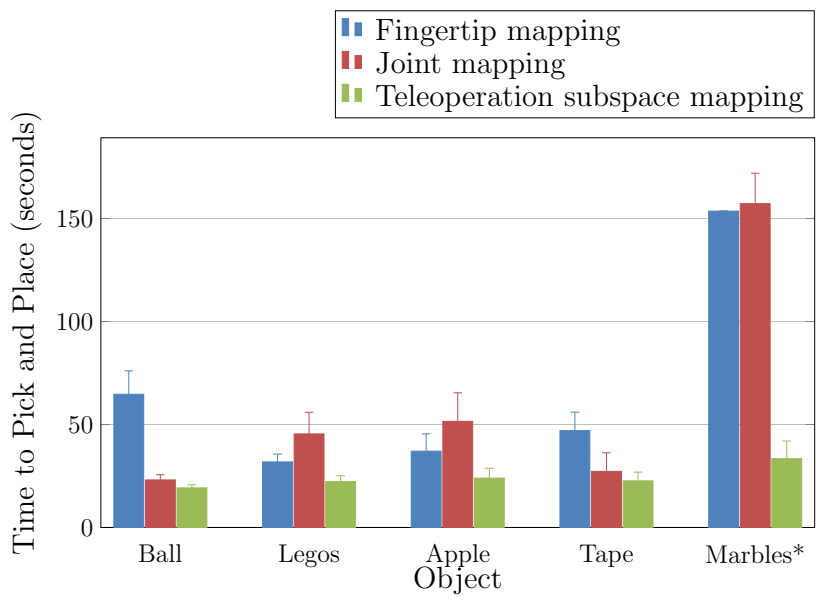

Fig. 7. Average time (in seconds) to pick and place objects using different teleoperation controls. * denotes that the average was calculated with a smaller sample size for some of the mapping methods of that object.

\section{E. Results}

We timed how long it took for the user to pick and place each object. If the user did not complete the task in four minutes, they were considered to be unable to pick up the object.

All five novices picked up the ball, legos, apple, and tape in the allotted time. All of the users picked up the marbles using subspace mapping. However, in the alloted time, only two users picked up the marbles using joint mapping and only one user picked up the marbles using fingertip mapping. We therefore calculated the average time to pick up the marbles from a smaller sample size for the fingertip and joint mappings (denoted by an asterisk in the results figures).

Table II and Figure 7 show the average time a novice took to pick and place an object using each of the control methods. We show our results numerically and graphically.

We found that novices using fingertip mapping took 2.75 times longer to pick and place an object than they took using teleoperation subspace mapping. On average, novice teleoperators using joint mapping took 2.51 times longer to complete a task than they took using subspace mapping.

For larger objects, like the ball and the tape, fingertip mapping took on average 2.75 times longer than subspace mapping, whereas joint mapping only took 1.2 times longer. For smaller objects, like the legos and the apple, fingertip mapping took 1.49 times longer than subspace mapping, whereas joint mapping took on average 2.09 times longer than subspace mapping.

\section{DISCUSSION}

The results show that teleoperation is possible using $T$ as an intermediary between the pose spaces of two dissimilar hands. This result has several meanings: first, it proves that our projection algorithm and our methods for calculating the projection, while simple, are sufficient to enable teleoperation. Second, it proves that $T$ is relevant to teleoperation for at least the human hand and the custom robotic hand used in our experiments. For these two hands, the teleoperation subspace encapsulates the range of motion needed to teleoperate the slave hand with the human hand.

Our experiments also show that teleoperation subspace mapping allows novice users to complete tasks more quickly than they are able to using either joint mapping or fingertip mapping. A novice user asked to pick up an object will, on average, complete the task 2.75 times slower using fingertip mapping and 2.51 times slower using joint mapping than they would using teleoperation subspace mapping. Using time to task completion as a metric for intuitiveness, these experiments prove that our subspace mapping is more intuitive for novice users than state of the art teleoperation mappings.

Fingertip mapping is applicable to precision grasps and joint mapping is applicable to power grasps [4]. Our experiments confirm this holds true for novice users. For larger objects, like the ball and the tape, which were selected to illustrate power grasps, joint mapping allowed the user to complete the task in half the time of fingertip mapping. For smaller objects, like the stack of Legos and the apple, which were selected to demonstrate precision grasps, the reverse was true - joint mapping took 1.43 times longer than fingertip mapping. Subspace mapping outperformed fingertip mapping and joint mapping for both precision grasps and power grasps, which shows that it is versatile enough to be applicable to different grasp types.

Overall, our experiments showed teleoperation subspace mapping to be faster and more versatile when presented with a variety of objects than state of the art mapping methods.

Our method makes several assumptions in order to teleoperate. Guided by postural synergies, we assume that the teleoperation subspace we have defined encapsulates all of the information needed to teleoperate a slave hand with only three basis vectors, and that mapping between joint space and teleoperation subspace is linear. We also assume that the user will be able to use intuition to generate the three variables needed for each hand to create the mapping from joint space to teleoperation subspace. In this work, we have provided a clear guideline so the user will be able to calculate these variables, and the process we describe is fairly simple. If the hand is so non-anthropomorphic that even human intuition cannot find a clear mapping into teleoperation subspace, our method will not apply.

Despite these assumptions, the potential applications of our method are broad. Unlike other teleoperation methods which use low dimensional subspaces, our method does not require the user to generate a large number of corresponding poses for the human and the robot. For each hand, the user 
must only provide three variables to enable teleoperation. In our method, the mapping between $T$ and pose space is independent of the master-slave pairing. If the mapping variables for multiple robots have been defined, then each human teleoperator only needs to provide the mapping for their specific hand (through a set of calibration poses) in order to teleoperate any of the robotic hands. It also is worth noting that, although we describe teleoperation in the context of a human controlling a robot, there is nothing in our method which requires this, and our method could theoretically be used to map poses between two robotic hands.

\section{COnClusions And Future Work}

In this paper, we propose an intuitive, low dimensional mapping between the pose spaces of the human hand and a non-anthropomorphic robot hand. We present teleoperation subspace as an intermediary between pose spaces of different hands. Projecting from pose space of the master hand into teleoperation subspace, and then projecting from teleoperation subspace into the pose space of the slave hand will enable teleoperation where the two hands make similar poses around a scaled object.

Our experiments show that the proposed teleoperation subspace is indeed relevant to teleoperation for at least two hands and that it can enable real-time teleoperation of a nonanthropomorphic hand. We also show that teleoperation subspace allows novice teleoperators to pick and place objects faster than state of the art teleoperation methods.

In the future, we would like to:

- Test our method with different robotic hands to see if teleoperation subspace is relevant to teleoperation for other hands with different kinematics.

- Automate building the projection between pose space and teleoperation subspace. Even though we present a simple method to calculate this projection, automating the process would make our method more accessible.

- Explore if the low dimensionality of teleoperation subspace can be leveraged to allow for teleoperation using different control inputs, like, for example, EMG signals.

- Study the sensitivity of the proposed mapping to changing parameters, such as different origin poses and joint maxima and minima.

\section{REFERENCES}

[1] M. Ferre, R. Aracil, C. Balaguer, M. Buss, and C. Melchiorri, Advances in telerobotics. Springer, 2007, vol. 31.

[2] M. V. Liarokapis, P. Artemiadis, C. Bechlioulis, and K. Kyriakopoulos, "Directions, methods and metrics for mapping human to robot motion with functional anthropomorphism: A review," School of Mechanical Engineering, National Technical University of Athens, Tech. Rep, 2013.

[3] I. Cerulo, F. Ficuciello, V. Lippiello, and B. Siciliano, "Teleoperation of the schunk s5fh under-actuated anthropomorphic hand using human hand motion tracking," Robotics and Autonomous Systems, vol. 89, pp. 75-84, 2017.

[4] R. Chattaraj, B. Bepari, and S. Bhaumik, "Grasp mapping for dexterous robot hand: A hybrid approach," in Contemporary Computing (IC3), International Conference on. IEEE, 2014, pp. 242-247.

[5] A. Peer, S. Einenkel, and M. Buss, "Multi-fingered telemanipulationmapping of a human hand to a three finger gripper," in Robot and Human Interactive Communication, 2008. RO-MAN 2008. The 17th IEEE International Symposium on. IEEE, 2008, pp. 465-470.
[6] R. N. Rohling, J. M. Hollerbach, and S. C. Jacobsen, "Optimized fingertip mapping: a general algorithm for robotic hand teleoperation," Presence: Teleoperators \& Virtual Environments, vol. 2, no. 3, pp. 203-220, 1993.

[7] W. B. Griffin, R. P. Findley, M. L. Turner, and M. R. Cutkosky, "Calibration and mapping of a human hand for dexterous telemanipulation," in ASME IMECE 2000 Symposium on Haptic Interfaces for Virtual Environments and Teleoperator Systems, 2000, pp. 1-8.

[8] H. Wang, K. H. Low, M. Y. Wang, and F. Gong, "A mapping method for telemanipulation of the non-anthropomorphic robotic hands with initial experimental validation," in Robotics and Automation, 2005. ICRA 2005. Proceedings of the 2005 IEEE International Conference on. IEEE, 2005, pp. 4218-4223.

[9] G. Gioioso, G. Salvietti, M. Malvezzi, and D. Prattichizzo, "Mapping synergies from human to robotic hands with dissimilar kinematics: an approach in the object domain," IEEE Transactions on Robotics, vol. 29, no. 4, pp. 825-837, 2013.

[10] G. Salvietti, L. Meli, G. Gioioso, M. Malvezzi, and D. Prattichizzo, "Object-based bilateral telemanipulation between dissimilar kinematic structures," in Intelligent Robots and Systems (IROS), 2013 IEEE/RSJ International Conference on. IEEE, 2013, pp. 5451-5456.

[11] _ "Multicontact bilateral telemanipulation with kinematic asymmetries," IEEE/ASME Trans. Mechatronics, vol. 22, no. 1, 2017.

[12] L. Pao and T. H. Speeter, "Transformation of human hand positions for robotic hand control," in Robotics and Automation. Proceedings., IEEE International Conference on. IEEE, 1989, pp. 1758-1763.

[13] S. Ekvall and D. Kragic, "Interactive grasp learning based on human demonstration," in Robotics and Automation. Proceedings. IEEE International Conference on, vol. 4. IEEE, 2004, pp. 3519-3524.

[14] T. Wojtara and K. Nonami, "Hand posture detection by neural network and grasp mapping for a master slave hand system," in Intelligent Robots and Systems, 2004.(IROS 2004). Proceedings. 2004 IEEE/RSJ International Conference on, vol. 1. IEEE, 2004, pp. 866-871.

[15] M. Santello, M. Flanders, and J. F. Soechting, "Postural hand synergies for tool use," J. Neurosci., vol. 18, no. 23, pp. 10 105-10 115, 1998.

[16] T. Feix, R. Pawlik, H.-B. Schmiedmayer, J. Romero, and D. Kragic, "A comprehensive grasp taxonomy," in Robotics, science and systems. workshop on understanding the human hand for advancing robotic manipulation, 2009, pp. 2-3.

[17] F. Ficuciello, G. Palli, C. Melchiorri, and B. Siciliano, "Planning and control during reach to grasp using the three predominant ub hand iv postural synergies," in Robotics and Automation (ICRA), 2012 IEEE International Conference on. IEEE, 2012, pp. 2255-2260.

[18] G. Palli, C. Melchiorri, G. Vassura, U. Scarcia, L. Moriello, G. Berselli, A. Cavallo, G. De Maria, C. Natale, S. Pirozzi, et al., "The dexmart hand: Mechatronic design and experimental evaluation of synergy-based control for human-like grasping," The International Journal of Robotics Research, vol. 33, no. 5, pp. 799-824, 2014.

[19] T. Geng, M. Lee, and M. Hülse, "Transferring human grasping synergies to a robot," Mechatronics, vol. 21, no. 1, pp. 272-284, 2011.

[20] O. C. Jenkins, "Sparse control for high-dof assistive robots," Intelligent Service Robotics, vol. 1, no. 2, pp. 135-141, 2008.

[21] A. Brygo, I. Sarakoglou, G. Grioli, and N. Tsagarakis, "Synergybased bilateral port: A universal control module for tele-manipulation frameworks using asymmetric master-slave systems," Frontiers in bioengineering and biotechnology, vol. 5, 2017.

[22] S. Kim, M. Kim, J. Lee, and J. Park, "Robot hand synergy mapping using multi-factor model and emg signal," in Experimental robotics. Springer, 2016, pp. 671-683.

[23] K. Yamane, Y. Ariki, and J. Hodgins, "Animating non-humanoid characters with human motion data," in Proceedings of the 2010 ACM SIGGRAPH/Eurographics Symposium on Computer Animation. Eurographics Association, 2010, pp. 169-178.

[24] A. Shon, K. Grochow, A. Hertzmann, and R. P. Rao, "Learning shared latent structure for image synthesis and robotic imitation," in Advances in neural information processing systems, 2006, pp. 1233-1240.

[25] B. Delhaisse, D. Esteban, L. Rozo, and D. Caldwell, "Transfer learning of shared latent spaces between robots with similar kinematic structure," in Neural Networks (IJCNN), 2017 International Joint Conference on. IEEE, 2017, pp. 4142-4149.

[26] J. Rosell, R. Suárez, and A. Pérez, "Safe teleoperation of a dual hand-arm robotic system," in ROBOT2013: First Iberian Robotics Conference. Springer, 2014, pp. 615-630. 